\title{
A-MUPS score to differentiate patients with somatic symptom disorder from those with medical disease for complaints of non-acute pain
}

This article was published in the following Dove Press journal: Journal of Pain Research

7 June 2017

Number of times this article has been viewed

\author{
Shingo Suzuki \\ Yoshiyuki Ohira \\ Kazutaka Noda \\ Masatomi Ikusaka \\ Department of General Medicine, \\ Chiba University Hospital, Chiba, \\ Japan
}

Correspondence: Shingo Suzuki Department of General Medicine, Chiba University Hospital, I-8-I, Inohana, Chuo-ku, Chiba 260-8677, Chiba, Japan $\mathrm{Tel} / \mathrm{Fax}+8 \mathrm{I} 432244758$

Email shngszkthk@yahoo.co.jp
Purpose: To develop a clinical score to discriminate patients with somatic symptom disorder (SSD) from those with medical disease (MD) for complaints of non-acute pain.

Methods: We retrospectively examined the clinical records of consecutive patients with pain for a duration of $\geq 1$ month in our department from April 2003 to March 2015. We divided the subjects according to the diagnoses of definite SSD (as diagnosed and tracked by psychiatrists in our hospital), probable SSD (without evaluation by psychiatrists in our hospital), matched MD (randomly matched two patients by age, sex, and pain location for each definite SSD patient), unmatched MD, other mental disease, or functional somatic syndrome (FSS). We investigated eight clinical factors for definite SSD and matched MD, and developed a diagnostic score to identify SSD. We subsequently validated the model with cases of probable SSD and unmatched MD.

Results: The number of patients with definite SSD, probable SSD, matched MD, unmatched MD, other mental disease, and FSS was 104 (3.5\%), 214 (7.3\%), 197 (6.7\%), 742 (25\%), 708 $(24 \%)$, and $978(33 \%)$, respectively. In a conditional logistic regression analysis, the following five factors were included as independent predictors of SSD: Analgesics ineffective, Mental disorder history, Unclear provocative/palliative factors, Persistence without cessation, and Stress feelings/episodes (A-MUPS). The area under the receiver operating characteristic curve (AUC) of the model was 0.900 (95\% CI: 0.864-0.937, $p<0.001$ ), and the McFadden's pseudo$R$-squared was 0.709 . For internal validation, the AUC between probable SSD and unmatched MD was 0.930 (95\% CI: $0.910-0.950, p<0.001)$. The prevalence and the likelihood ratio of SSD increased as the score increased.

Conclusion: The A-MUPS score was useful for discriminating patients with SSD from those with MD for complaints of non-acute pain, although external validation and refinement should be needed.

Keywords: medically unexplained, somatoform, somatization, hypochondriasis, pain disorder, functional somatic syndrome

\section{Introduction}

Somatic symptom disorder (SSD) is conceptualized as a psychiatric disorder, which shows an excess degree of suffering beyond that resulting from the presence of somatic symptoms alone, as defined in the Diagnostic and Statistical Manual of Mental Disorders (DSM), Fifth Edition. ${ }^{1}$ The diagnostic criteria consist of the following: one or more somatic symptoms that disrupt patients' daily life (criterion A); excessive thoughts, feelings, or behaviors related to their symptoms (criterion B); and a symptomatic state that typically persists for more than six months (criterion C). ${ }^{2} \mathrm{SSD}$ encompasses somatization disorder, undifferentiated somatoform disorder (SFD), pain disorder, and hypochondriasis (with somatic symptoms) of SFD as defined in DSM-IV, ${ }^{1}$ although 
excessive inclusion of patients is a concern, ${ }^{3}$ and can be diagnosed with or without the presence of medical disease (MD). ${ }^{2}$ In this study, because these various names may be confusing, we use the term SSD to encompass all the terms that are included in the concept of SSD.

SSD is common because somatization accounts for $10 \%$ of primary care patients, which is the same prevalence as depression and anxiety. ${ }^{4}$ However, because SSD is one of the most difficult diseases to diagnose,${ }^{5}$ doctors may order unnecessary diagnostic procedures to avoid overlooking MD. ${ }^{6}$ Such excessive interventions can foster somatic fixations of patients, ${ }^{7}$ leading to functional impairment, higher disability days, and increased health care costs. ${ }^{4}$ In addition, the early detection of mental illness is critical even for patients with somatic disease because mental treatments are recommended as soon as possible so as to prevent chronic state. ${ }^{8}$ Thus, there is a need to develop a comprehensive biopsychosocial approach that may be applied at an early stage. ${ }^{6}$

However, no gold standard diagnostic method has been established for SSD. ${ }^{9}$ Even the diagnostic criteria of the DSM-5 necessitate clinical experience when making assessments. ${ }^{10}$ Moreover, previous studies have used various terms for medically unexplained physical symptoms (MUPS), such as somatization, SFD, and functional somatic symptom, ${ }^{11}$ and these various definitions and methods might have led to inconsistent conclusions. ${ }^{12}$ Thus, SSD and its clinical features in particular have scarcely been studied. ${ }^{13}$

SSD patients may complain of various symptoms including pain, fatigue, and issues pertaining to gastrointestinal, cardiopulmonary, urogenital, neurological, skin and glands, or autonomic systems. ${ }^{14}$ Here, we studied patients with pain, which is common but of which it is challenging for doctors to determine the causes. Our objective was to develop a clinical score for discriminating patients with SSD from those with MD because physicians frequently are unable to identify the principal cause of pain between the two types of disorders, and worry about overlooking MD. We do not encourage mind-body dualism, and one reason for "excluding MD", as abolished in the DSM-5, is related to limited reliability for determining patients' symptoms as MUPS. ${ }^{2}$ Accordingly, clinical features of SSD were compared to those of MD to develop our clinical score.

\section{Methods}

\section{Study population}

This was a retrospective cross-sectional study conducted at a university hospital in Japan. We investigated the medical records of consecutive patients who visited the Department of General Medicine at Chiba University Hospital with pain from April 2003 to March 2015. We list the classification of patients in Figure 1. Because the purpose of this study was to investigate differences in clinical features between SSD and MD, we excluded patients complaining of acute pain, which doctors rarely consider as SSD. We defined acute pain as that with a duration of less than one month since onset because acute pain generally improves within several weeks. ${ }^{15}$ Patients were also excluded if their final diagnoses were undetermined or if we could not understand them in sufficient detail due to language problems, dementia, an uncooperative attitude, mental retardation, or deafness. Because the clinical features of mental disorders other than SSD, such as depression or anxiety, have been established and these disorders contribute independently to functional impairment, ${ }^{4}$ such disorders should be evaluated before considering whether a patient might be suffering from SSD in primary care settings. Therefore, patients with a chief diagnosis of a mental disorder other than SSD, including those comorbid with SSD, were also excluded.

\section{Confirmation of diagnoses}

Our department has a system whereby two or more physicians examine each patient, and spend sufficient time to evaluate biopsychosocial problems, because most patients visiting our department are referred with unknown diagnostics from primary or secondary medical institutions. If a diagnosis is inconsistent or unclear among the physicians, they consult another senior physician or specialist, or follow up until a final diagnosis is determined. Diagnoses of mental disorder were previously based on the DSM-IV and are currently based on the DSM-5, or by referral to psychiatrists in daily medical practice. When extracting the data for this study, two physicians independently checked the clinical records to determine the final diagnosis (thus, a total of four or more physicians confirmed each diagnosis), and the diagnoses of mental disorder, including SSD, were reconfirmed based on the DSM-5. If the final diagnosis was in doubt, further discussion took place with the physicians in charge of the patient. However, because there is no widely accepted method to diagnose SSD, ${ }^{9}$ reliability of the diagnosis is an important problem. We classified patients as having "definite SSD" for developing a diagnostic score based on confirmed diagnosis of SSD if two or more physicians and a psychiatrist in our hospital diagnosed SSD with no alteration of diagnosis after follow-up. Patients were classified as having "probable SSD" for validating the model if the diagnosis was by physicians without an evaluation by psychiatrists in our hospital, even if 


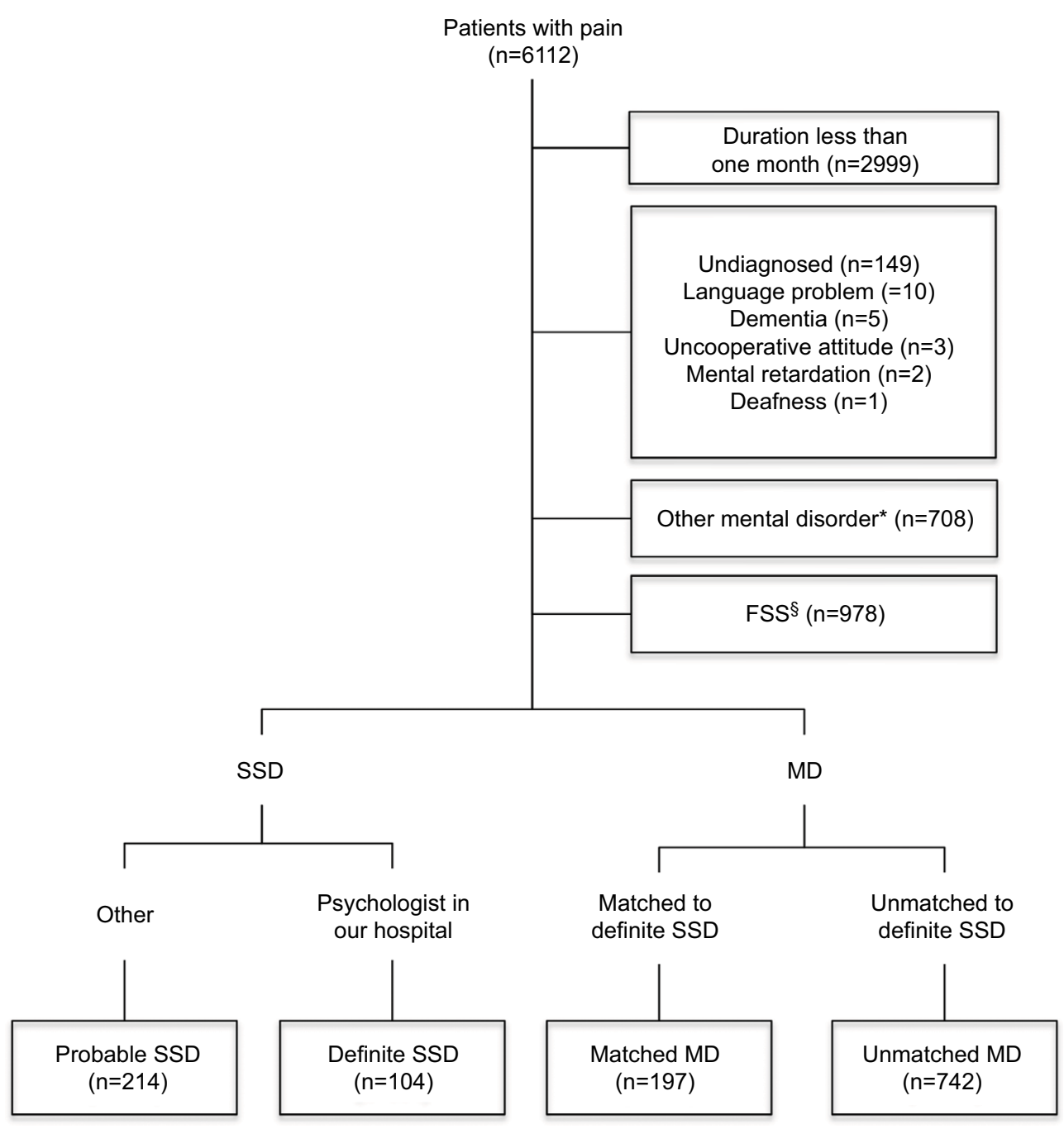

Figure I Flowchart and classification of the patients.

Notes: *Including SSD if the primary diagnosis was another mental disorder. ${ }^{\S}$ Not combined with the diagnosis of SSD.

Abbreviations: SSD, somatic symptom disorder; FSS, functional somatic syndrome; MD, medical disease.

there existed a consistent diagnosis made by psychiatrists in other institutions. Although the concept of functional somatic syndrome (FSS) overlaps with SSD, patients with FSS who also meet SSD criteria have greater disability than those who do not, ${ }^{9}$ indicating that FSS can be regarded as a mild disorder compared to SSD. Thus, we classified patients as having "FSS" if they complained of nonspecific pain, as well as if they had a diagnosis of FSS, such as irritable bowel disease, non-ulcer dyspepsia, premenstrual syndrome, chronic pelvic pain, fibromyalgia, atypical or noncardiac chest pain, chronic fatigue syndrome, tension headache, temporomandibular joint dysfunction, or atypical facial pain, ${ }^{11}$ when they did not meet the diagnostic criteria for SSD according to the DSM-5. Patients who met the criteria for both SSD and FSS were classified into the SSD group because most of such patients needed to be referred to psychiatrists, unlike cases of FSS only. However, the clinical position of FSS is unclear, ${ }^{13}$ in terms of whether it should be categorized within SSD or strictly distinguished as non-SSD. Moreover, this study aimed to develop a clinical score to discriminate SSD from MD, and physicians who diagnose patients with FSS-related disease based on their diagnostic criteria but omit the diagnosis of SSD would not strengthen the patients' somatic fixations because they are not concerned with MD; rather, they target functional improvement in such conditions. Therefore, we excluded FSS when developing our model, but investigated its nature in the validation phase.

\section{Procedure}

We randomly matched two patients with MD (defined as "matched MD") by age (within two years), sex, and pain location (at least one part) for each definite SSD patient 
to exclude biases related to these factors and to generate a versatile model because the diagnoses and clinical features of MD differ according to these factors. The pain locations were divided into eight: whole body (if patients used the term or if parts included all the upper and lower limbs, and trunk), head and neck, chest, abdomen, back, upper limb, lower limb, and genital area. We decided upon eight clinical factors to investigate via focus-group discussions in our department, based on past reports and clinical experiences: analgesics ineffective (we defined analgesics as only acetaminophen or nonsteroidal anti-inflammatory drugs [NSAIDs]), unclear provocative/palliative factors, persistence without cessation, progression, mental disorder history, stress feelings/episodes (prior to the onset or aggravation of pain), complaints other than pain, and duration of six or more months (from the onset of pain to the first visit to our department). Two physicians independently checked each clinical record to extract the data. When the data were inconsistent, this was resolved by discussion with a third physician. Although all factors were evaluated by interview sheet or medical record history, unclear provocative/palliative factors were also evaluated by physical examination.

In the validation, probable SSD patients and MD patients who were unmatched to definite SSD patients (defined as "unmatched MD") were employed. All cases of SSD and all cases of MD were also employed because each SSD and MD group was not divided randomly, to prioritize confirming diagnoses of SSD in developing a model. To evaluate the nature of FSS, we assessed FSS + all SSD versus all MD, and all SSD versus FSS + all MD. Study approval was obtained from the institutional review board of the Graduate School of Medicine, Chiba University, without requirement of patient written consent because this study included no information that can reveal the identity of a particular individual, thus ensuring confidentiality of patient data.

\section{Statistical analysis}

In the derivation, the factor of analgesics ineffective was evaluated according to 11 grades of improvement (0 [no effect] to 10 [temporarily complete disappearance of pain]). Missing data for this factor were substituted by the mean value of the two groups. We subsequently decided a binary cut-off value via the area under the receiver operating characteristic curve (AUC). Subsequently, the eight clinical factors of relevance to definite SSD and matched MD were evaluated by univariate analyses. The factors with a $P$-value of less than 0.15 in the univariate analyses were included in a conditional logistic regression model with forced entry method to identify the significant predictors $(p<0.05)$. The description utility of the model was evaluated by AUC, and the goodness of fit of the model was assessed using McFadden's pseudo- $R$-squared.

In the internal validation, the discrimination ability was assessed via AUC. The calibration was assessed by comparing the prevalence and the likelihood ratio of SSD according to the risk score. We regarded missing data for each factor as negative. We used StatsDirect version 3 (StatsDirect Ltd., Altricham, UK) for calculating the odds ratios for univariate analyses and SPSS Statistics for Windows version 22 (IBM Corp., Armonk, NY, USA) for other statistical analyses.

\section{Results}

\section{Characteristics of the participants}

We show age, sex, and pain locations for each group in Table 1 and the final diagnoses of MD according to pain location in the Supplementary material. There were 104 cases of definite SSD and 197 cases of matched MD (Figure 1).

Table I Age, gender, and pain location in each group

\begin{tabular}{|c|c|c|c|c|c|c|c|}
\hline \multirow{2}{*}{$\begin{array}{l}\text { Basic } \\
\text { information }\end{array}$} & \multicolumn{3}{|l|}{ Derivation } & \multicolumn{4}{|l|}{ Validation } \\
\hline & $\begin{array}{l}\text { Definite SSD, } \\
n=104\end{array}$ & $\begin{array}{l}\text { Matched MD, } \\
n=197\end{array}$ & $P$-value & $\begin{array}{l}\text { Probable SSD, } \\
n=2 \text { | } 4\end{array}$ & $\begin{array}{l}\text { Unmatched MD, } \\
n=742\end{array}$ & $\begin{array}{l}\text { FSS, } \\
n=978\end{array}$ & p-value \\
\hline Age & $52.0(16.6)$ & $52.1(16.4)$ & 0.97 & $47.3(16.5)$ & $54.2(18.0)$ & 48.7 (18.1) & $<0.001$ \\
\hline Male & 42 (40\%) & 79 (40\%) & 0.96 & 69 (32\%) & 345 (46\%) & 437 (45\%) & 0.009 \\
\hline \multicolumn{8}{|l|}{ Location* } \\
\hline Whole body & $16(15 \%)$ & 28 (14\%) & 0.78 & 57 (27\%) & 41 (6\%) & $39(4 \%)$ & $<0.001$ \\
\hline Head, neck & 35 (34\%) & $46(23 \%)$ & 0.055 & $53(25 \%)$ & $120(16 \%)$ & 297 (30\%) & $<0.001$ \\
\hline Chest & $20(19 \%)$ & $30(15 \%)$ & 0.38 & 15 (7\%) & $106(14 \%)$ & 154 (I6\%) & 0.004 \\
\hline Abdomen & $29(28 \%)$ & $5 I(26 \%)$ & 0.71 & $46(21 \%)$ & |3| (18\%) & $319(33 \%)$ & $<0.001$ \\
\hline Back & 21 (20\%) & 38 (19\%) & 0.85 & $42(20 \%)$ & 97 (I3\%) & 184 (19\%) & 0.003 \\
\hline Upper limb & 14 (I3\%) & $22(11 \%)$ & 0.56 & $22(10 \%)$ & 155 (21\%) & $61(6 \%)$ & $<0.001$ \\
\hline Lower limb & II (II\%) & $30(15 \%)$ & 0.26 & $36(17 \%)$ & $229(31 \%)$ & 70 (7\%) & $<0.001$ \\
\hline Genital area & $4(4 \%)$ & $4(2 \%)$ & 0.45 & $6(3 \%)$ & $6(1 \%)$ & $24(2 \%)$ & 0.025 \\
\hline
\end{tabular}

Notes: Data are mean (SD) or $\mathrm{n}(\%)$. *Multiple locations could be chosen, except in the case of whole body, if patients suffered pain in multiple regions.

Abbreviations: SSD, somatic symptom disorder; MD, medical disease; FSS, functional somatic syndrome. 
Definite SSD cases that matched only one MD patient due to the lack of a matching case meeting all conditions consisted of the following, listed by pain location: four whole body (18-, 39-, 59-, and 68-year-old females), one head and neck (55-year-old female), one chest (65-year-old female), one back (38-year-old male), one upper limb (23-year-old female), and three genital area (60-year-old female and 61- and 66-year-old males). The number of cases of probable SSD, unmatched MD, and FSS was 214, 742, and 978, respectively (Figure 1).

\section{Derivation}

Pain improvement according to 11 grades of analgesia was recorded in $59(57 \%)$ cases of definite SSD and $82(42 \%)$ cases of matched MD. Missing values were substituted with the mean value of 2.3, while effectiveness (including no record of concrete improvement grades), ineffectiveness, and missing data occurred in six (5.8\%), 56 (54\%), and 42 (40\%) cases of definite SSD and 79 (40\%), 32 (16\%), and $86(44 \%)$ cases of matched MD, respectively. The cut-off score was determined to be between 0 and 1 or more $(\mathrm{AUC}=0.789 ; 95 \%$ CI: $0.714-0.863, p<0.001)$. All factors, except for progression, had a $p$-value of less than 0.15 in univariate analyses (Table 2). In the conditional logistic regression analysis with forced entry method, the following five factors were included as independent predictors of SSD $(p<0.05)$ in decreasing order of odds ratios (Table 3): mental disorder history, unclear provocative/palliative factors, analgesics ineffective, stress feelings/episodes, and persistence without cessation. The AUC of the model was 0.900 (95\% CI: 0.864-0.937, $p<0.001$; Figure 2), and the McFadden's pseudo- $R$-squared was 0.709 .

\section{Validation}

We show the results of five factors for probable SSD, unmatched MD, and FSS in Table 4. AUC was 0.930 (95\% CI: $0.910-0.950, p<0.001$ ) for probable SSD versus unmatched

Table 2 Univariate analysis of the eight factors between definite SSD and matched MD

\begin{tabular}{|c|c|c|c|c|c|}
\hline Factors & Definite SSD, $n=104$ & Matched MD, n= I 97 & Odds ratio & $95 \% \mathrm{Cl}$ & $p$-value \\
\hline \multicolumn{6}{|l|}{ Analgesics* } \\
\hline Effective & 48 (46\%) & I 65 (84\%) & Ref & & \\
\hline Ineffective & $56(54 \%)$ & $32(16 \%)$ & 6.02 & $3.39-10.7$ & $<0.001$ \\
\hline Missing data & $0(0 \%)$ & $0(0 \%)$ & NA & NA & NA \\
\hline \multicolumn{6}{|c|}{ Provocative/palliative factors } \\
\hline Clear & $33(32 \%)$ & 153 (78\%) & Ref & & \\
\hline Unclear & $71(68 \%)$ & $43(22 \%)$ & 7.66 & $4.34-13.5$ & $<0.001$ \\
\hline Missing data & $0(0 \%)$ & $\mathrm{I}(<\mathrm{I} \%)$ & NA & NA & NA \\
\hline \multicolumn{6}{|l|}{ Persistence } \\
\hline With cessation & $30(29 \%)$ & 131 (66\%) & Ref & & \\
\hline Without cessation & $73(70 \%)$ & $63(32 \%)$ & 5.06 & $2.02-8.84$ & $<0.001$ \\
\hline Missing data & I (I\%) & $3(2 \%)$ & 1.46 & $0.03-18.8$ & 0.57 \\
\hline \multicolumn{6}{|l|}{ Progression } \\
\hline Progressing & $36(35 \%)$ & $55(28 \%)$ & Ref & & \\
\hline Nonprogressing & 64 (62\%) & | 40 (7|\%) & 0.70 & $0.4|-| .2 \mid$ & 0.18 \\
\hline Missing data & $4(4 \%)$ & $2(1 \%)$ & 3.06 & $0.4 I-35.0$ & 0.23 \\
\hline \multicolumn{6}{|l|}{ Mental disorder history } \\
\hline Existent & $20(19 \%)$ & $9(5 \%)$ & 4.91 & $2.02-12.7$ & $<0.001$ \\
\hline Nonexistent & $81(78 \%)$ & 179 (9l\%) & Ref & & \\
\hline Missing data & $3(3 \%)$ & $9(5 \%)$ & 0.74 & $0.13-3.06$ & 0.76 \\
\hline \multicolumn{6}{|l|}{ Stress feelings/episodes } \\
\hline Existent & $55(53 \%)$ & $33(17 \%)$ & 5.67 & $3.13-10.3$ & $<0.001$ \\
\hline Nonexistent & $40(38 \%)$ & 136 (69\%) & Ref & & \\
\hline Missing data & $9(9 \%)$ & $28(14 \%)$ & 1.09 & $0.42-2.63$ & 0.83 \\
\hline \multicolumn{6}{|c|}{ Complaints other than pain } \\
\hline Existent & $58(56 \%)$ & $64(32 \%)$ & 2.62 & $1.56-4.40$ & $<0.001$ \\
\hline Nonexistent & $46(44 \%)$ & $133(68 \%)$ & Ref & & \\
\hline Missing data & $0(0 \%)$ & $0(0 \%)$ & NA & NA & NA \\
\hline \multicolumn{6}{|l|}{ Duration } \\
\hline Six or more months & $71(68 \%)$ & 107 (54\%) & 1.87 & $1.10-3.20$ & 0.019 \\
\hline Under six months & $32(31 \%)$ & $90(46 \%)$ & Ref & & \\
\hline Missing data & I (I\%) & $0(0 \%)$ & NA & NA & NA \\
\hline
\end{tabular}

Note: *Missing data were substituted by the mean value of the two groups.

Abbreviations: SSD, somatic symptom disorder; MD, medical disease; Ref, reference; NA, not applicable. 
Table 3 Conditional logistic regression analysis of the factors with $p<0.15$ in univariate analysis

\begin{tabular}{lllll}
\hline Factors & Regression $\boldsymbol{\beta}$ coefficient & Odds ratio & $\mathbf{9 5 \%} \mathbf{C l}$ & $\mathbf{p}$-value \\
\hline Mental disorder history & 2.64 & 14.1 & $3.49-56.6$ & $<.6 .001$ \\
Unclear provocative/palliative factor & 2.32 & 10.2 & $3.71-28.1$ & $<0.001$ \\
Analgesics ineffective & 2.28 & 9.73 & $3.44-27.4$ & $<0.001$ \\
Stress feelings/episodes & 2.12 & 8.29 & $2.69-25.5$ & $<0.001$ \\
Persistence without cessation & 1.90 & 6.69 & $2.25-19.9$ & 0.001 \\
Complaints other than pain & 0.85 & 2.35 & $0.91-6.06$ & 0.078 \\
Duration of six or more months & 0.65 & 1.92 & $0.83-4.44$ & 0.13 \\
\hline
\end{tabular}

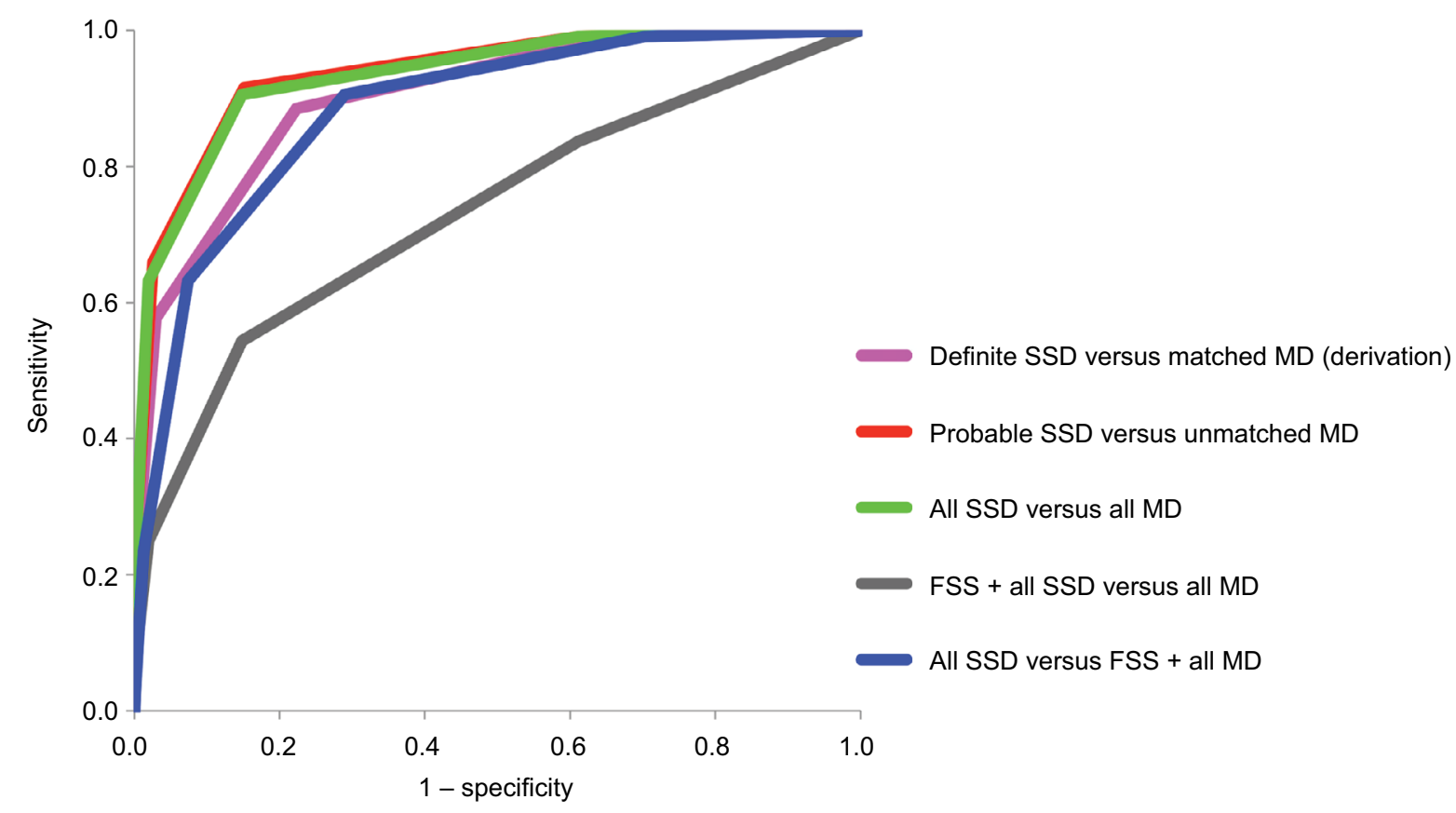

Figure 2 Receiver operating characteristic curves according to the five scores in derivation and validation phases. Abbreviations: SSD, somatic symptom disorder; MD, medical disease; FSS, functional somatic syndrome.

MD, 0.932 (95\% CI: 0.916-0.949, $p<0.001)$ for all SSD versus all MD, 0.730 (95\% CI: $0.710-0.751, p<0.001)$ for all SSD + FSS versus all MD, and 0.881 (95\% CI: 0.862-0.901, $p<0.001$ ) for all SSD versus all MD + FSS (Figure 2). The prevalence and the likelihood ratio of SSD increased as the score increased (Figures 3-6). When we defined the cut-off score as between 1 and 2, corresponding to the largest sum of sensitivity and specificity, the score of the five factors had a sensitivity of 0.916 and a specificity of 0.849 for probable SSD versus unmatched MD, 0.906 and 0.852 for all SSD versus all MD, 0.543 and 0.852 for all SSD + FSS versus all MD, and 0.906 and 0.710 for all SSD versus all MD + FSS, respectively.

\section{Discussion}

This is the first study to reveal the clinical features of SSD compared to $\mathrm{MD}$, and to suggest a diagnostic score for nonacute pain. The following five factors composed the score:
Analgesics ineffective, Mental disorder history, Unclear provocative/palliative factors, Persistence without cessation, and Stress feelings/episodes (A-MUPS). Based on the discrimination ability of the A-MUPS score according to AUC (Figure 2) and the stratified prevalence and likelihood ratio of SSD in the validation (Figures 3-6), the clinical features of FSS are more similar to those of MD than those of SSD. This indicates that the A-MUPS score reflects specific clinical features of SSD defined in the DSM-5, that it would be possible to assess whether patients with FSS had a diagnosis combined with SSD or not, and that the concept of SSD should be separated from that of FSS.

Factor "A" of the A-MUPS score was Analgesics ineffective. Acetaminophen and NSAIDs are recommended in the initial treatment for cancer and non-cancer pain. ${ }^{15}$ Besides pharmacological mechanisms, placebo analgesia, to which endogenous opioids and dopamine contribute, influences analgesic effects. ${ }^{16}$ Positive expectancies strengthen the 
degree of the placebo effect, while negative expectancies decrease the placebo effect, increase side effects, and induce nocebo effects. ${ }^{17}$ Although SSD patients visit multiple doctors as they seek care, the purpose of their behavior tends to be to persuade others that they are ill, so as to obtain

Table 4 The five factors for probable SSD, unmatched MD, and FSS in the validation phase

\begin{tabular}{|c|c|c|c|}
\hline Factors & $\begin{array}{l}\text { Probable SSD, } \\
n=214\end{array}$ & $\begin{array}{l}\text { Unmatched } \\
\text { MD, } n=742\end{array}$ & $\begin{array}{l}\text { FSS, } \\
n=978\end{array}$ \\
\hline \multicolumn{4}{|l|}{ Analgesics } \\
\hline Effective & $25(12 \%)$ & $264(36 \%)$ & $188(19 \%)$ \\
\hline Ineffective & $106(50 \%)$ & $99(13 \%)$ & $15 \mathrm{I}(15 \%)$ \\
\hline Missing data & $83(39 \%)$ & $379(51 \%)$ & $639(65 \%)$ \\
\hline \multicolumn{4}{|c|}{ Provocative/palliative factors } \\
\hline Clear & $41(19 \%)$ & $593(80 \%)$ & $520(53 \%)$ \\
\hline Unclear & $|7|(80 \%)$ & 145 (20\%) & 445 (46\%) \\
\hline Missing data & $2(<1 \%)$ & $4(<1 \%)$ & $13(1 \%)$ \\
\hline \multicolumn{4}{|l|}{ Persistence } \\
\hline With cessation & $56(26 \%)$ & $493(66 \%)$ & 699 (7I\%) \\
\hline Without cessation & $157(73 \%)$ & $240(32 \%)$ & $264(27 \%)$ \\
\hline Missing data & $\mathrm{I}(<\mathrm{I} \%)$ & $9(1 \%)$ & $15(2 \%)$ \\
\hline \multicolumn{4}{|c|}{ Mental disorder history } \\
\hline Existent & $68(32 \%)$ & $45(6 \%)$ & III (II\%) \\
\hline Nonexistent & $14 \mid(66 \%)$ & $671(90 \%)$ & $812(83 \%)$ \\
\hline Missing data & $5(2 \%)$ & $26(4 \%)$ & $54(6 \%)$ \\
\hline \multicolumn{4}{|c|}{ Stress feelings/episodes } \\
\hline Existent & II 3 (53\%) & $58(8 \%)$ & $364(37 \%)$ \\
\hline Nonexistent & 93 (43\%) & 544 (73\%) & $490(50 \%)$ \\
\hline Missing data & $8(4 \%)$ & $130(18 \%)$ & 124 (13\%) \\
\hline
\end{tabular}

Abbreviations: SSD, somatic symptom disorder; MD, medical disease; FSS, functional somatic syndrome. illness-related privileges. ${ }^{13}$ These properties may contribute to the ineffectiveness of analgesics.

Factor "M" was Mental disorder history. The comorbidity of SSD with depression and/or anxiety is high, with a rate of $26 \%-59 \%,{ }^{18}$ and patients with mental disorder tend to develop SSD. ${ }^{19}$ In a study of anxiety disorder, the proportion of cases with SSD that followed anxiety versus anxiety that followed SSD was the same, and simultaneous onset of these disorders was rare. ${ }^{20}$ Mental disorders influence each other.

Factor "U" was Unclear provocative/palliative factors. SSD patients complain of vague, imprecise, and changeable physical symptoms. ${ }^{21}$ Diagnostic criteria for SSD in the DSM-5 include "excessive thoughts, feelings, or behaviors related to somatic symptoms (criterion B)"; ${ }^{2}$ functional, cortical issues would evoke or strengthen such symptoms. It is likely that no or minimal pain signal arises from the somatic organs, and thus, provocative/palliative factors cannot be clear.

Factor "P" was Persistence without cessation. Diagnostic criteria for SSD in the DSM-5 include persistence of symptoms. ${ }^{2}$ Although somatic symptoms need not be continuous,${ }^{22}$ our study showed that the possibility of SSD increased if pain continued without cessation. Because SSD patients have a tendency of selective attention to physical symptoms,${ }^{18}$ unceasing consciousness of their pain would be causative.

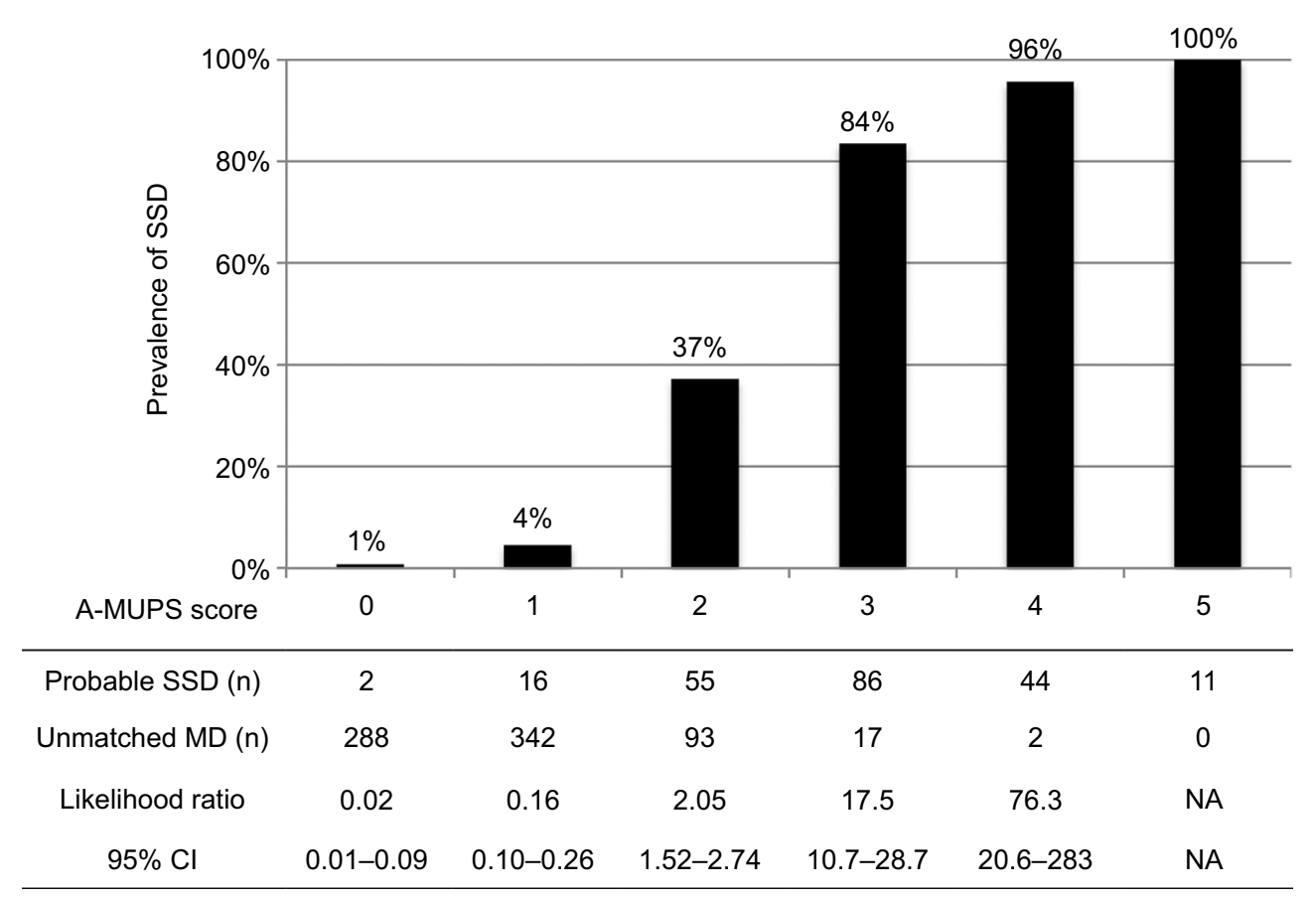

Figure 3 Prevalence and likelihood ratio of SSD in validation phase (probable SSD versus unmatched MD).

Abbreviations: A-MUPS, Analgesics ineffective, Mental disorder history, Unclear provocative/palliative factors, Persistence without cessation, and Stress feelings/episodes; SSD, somatic symptom disorder; MD, medical disease; NA, not applicable. 


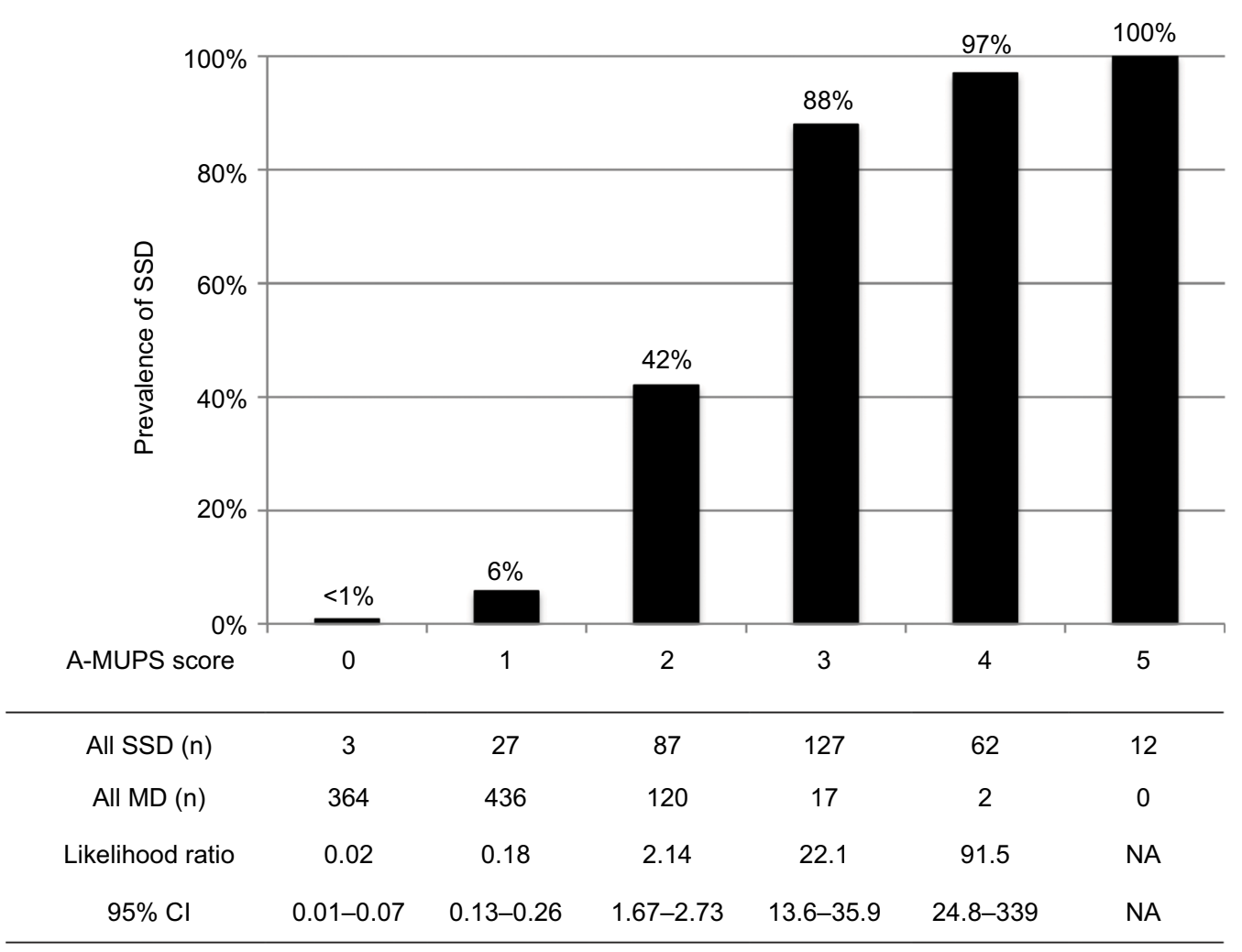

Figure 4 Prevalence and likelihood ratio of SSD in validation phase (all SSD versus all MD).

Abbreviations: A-MUPS, Analgesics ineffective, Mental disorder history, Unclear provocative/palliative factors, Persistence without cessation, and Stress feelings/episodes; SSD, somatic symptom disorder; MD, medical disease; NA, not applicable.

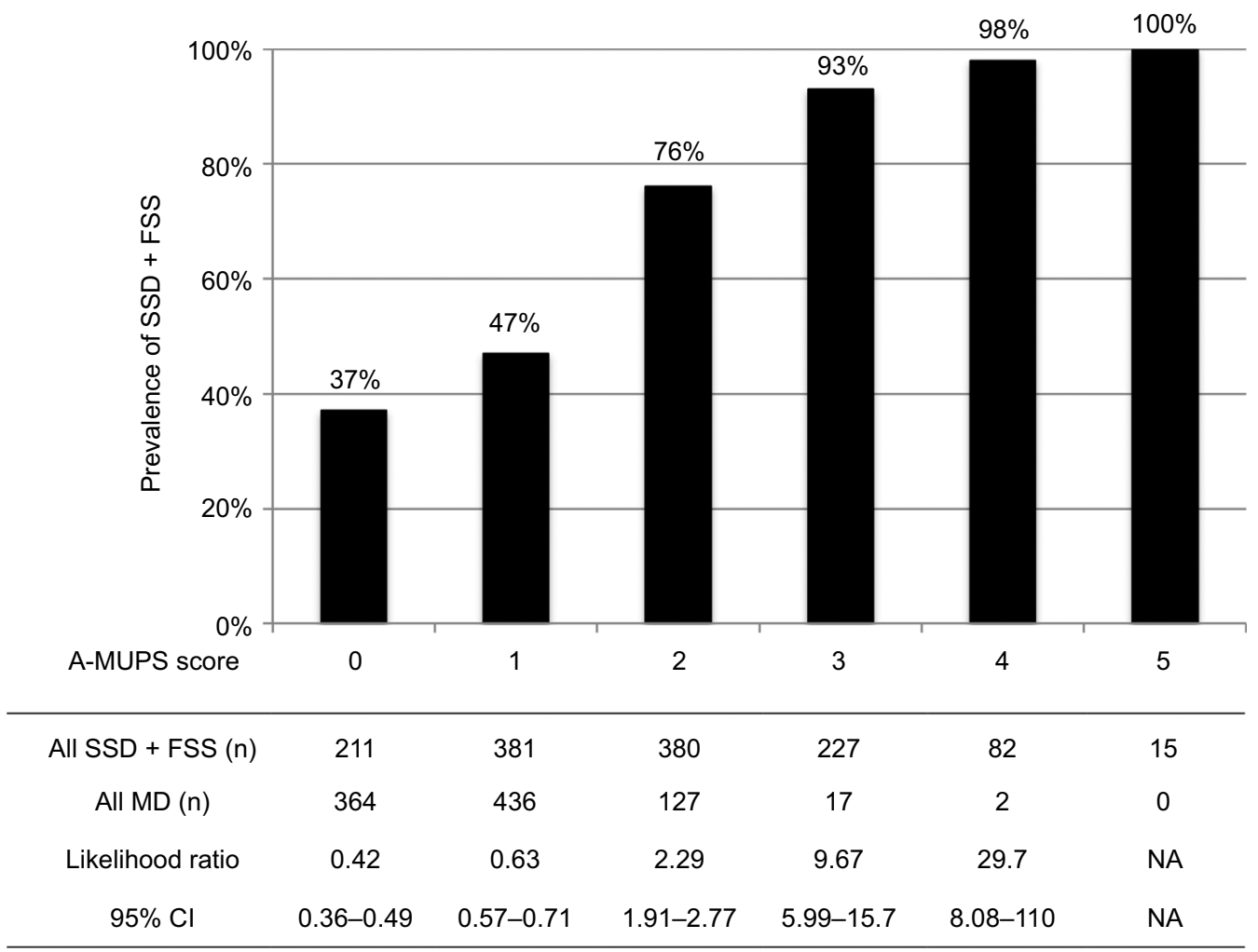

Figure 5 Prevalence and likelihood ratio of SSD + FSS in validation phase (all SSD + FSS versus all MD).

Abbreviations: A-MUPS, Analgesics ineffective, Mental disorder history, Unclear provocative/palliative factors, Persistence without cessation, and Stress feelings/episodes; SSD, somatic symptom disorder; FSS, functional somatic syndrome; MD, medical disease; NA, not applicable. 


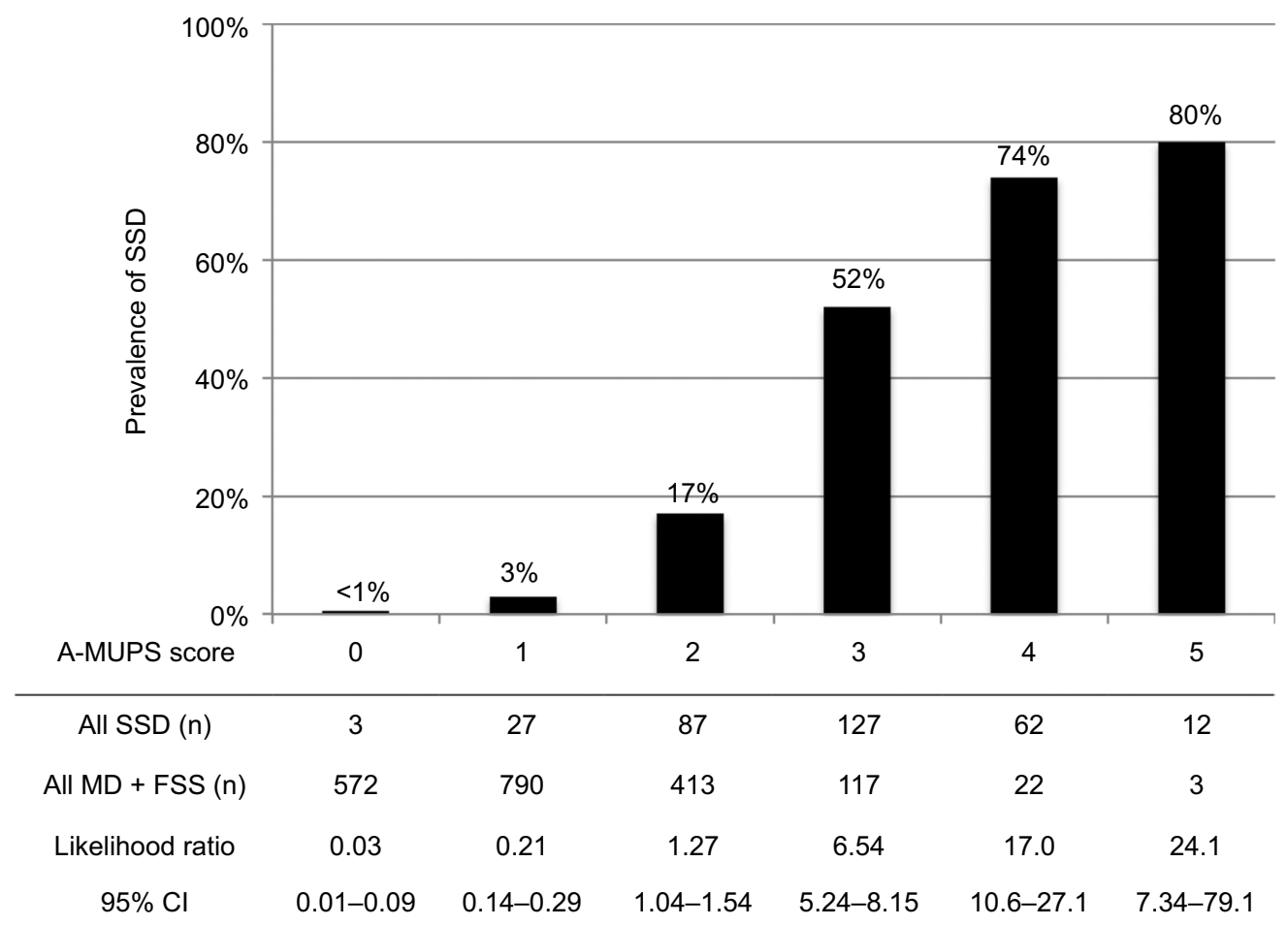

Figure 6 Prevalence and likelihood ratio of SSD in validation phase (all SSD versus all MD + FSS).

Abbreviations: A-MUPS, Analgesics ineffective, Mental disorder history, Unclear provocative/palliative factors, Persistence without cessation, and Stress feelings/episodes; SSD, somatic symptom disorder; MD, medical disease; FSS, functional somatic syndrome.

Factor "S" was stress feelings/episodes. Mental pain share a common neuroanatomical pathway with physical pain; the anterior cingulate cortex is related to the experience of pain distress, and the right ventral prefrontal cortex is related to the regulation of pain distress. ${ }^{23}$ Thus, cortical processing and interpretation of somatic signals depend on psychological salience, ${ }^{24}$ so that cognition, mood, and context influence pain perception. ${ }^{25}$ As SSD patients are more susceptible to pain than healthy individuals who are experiencing transient negative affect,${ }^{26}$ the former are readily affected by stress.

SSD patients often complain of multiple symptoms, ${ }^{14}$ but pain tends to be the sole severe complaint. ${ }^{2}$ Although this is a reason why the factor "complaints other than pain" was not included in the model, some MD patients had objectively and easily identifiable complaints, including fever, swelling, and pallor. If we considered the quality of complaints, the factor may become of utility. Although the typical duration of symptoms is usually considered as more than six months, the factor "duration of six or more months" was also not included in the model. This suggests that SSD can be detected at an early stage, via other clinical features assessed by A-MUPS. Progression of pain generally indicates severe MD, while SSD patients attempt to convince others of their severe symptoms claiming aggravation. ${ }^{27}$ Thus, it is difficult to distinguish SSD from MD via the time course of pain.

\section{Strengths and limitations of this study}

A strength of this study is its utilization of consecutive patients, most of whom were referred with unknown diagnostics. In addition, diagnoses were confirmed by two or more physicians who spent sufficient time to reach a satisfactory diagnosis, and diagnoses were also independently checked by another two physicians to minimize misdiagnosis. Moreover, we used the definite SSD data, which incorporated diagnosis by psychiatrists in our hospital with follow-up, to formulate our model. Although the results of the validation suggest the model is useful, these methods might have led to sampling bias.

Our study has several limitations. The possibility of sampling bias should be noted because the setting of this study was a single university hospital in Japan. Although our diagnoses of mental disorder including SSD were reconfirmed based on DSM- 5 criteria by two or more independent physicians, they relied on medical records but not the medical practice itself. In addition, this was a retrospective crosssectional study, although we tried to prevent recall bias and observer bias by double independent evaluations of medical records. Considering these limitations, we need to obtain prospective external validations in other multicenter settings. Mean-value substitution was used to replace missing data for the factor "analgesics ineffective" while generating the 
model because the proportion of missing data was high due to no prior prescription, absence of history, or the restricted definition of analgesics as those initially recommended (i.e., acetaminophen and NSAIDs). ${ }^{15}$ However, in the model formulation, the proportions of effectiveness (including no record of concrete improvement grades), ineffectiveness, and missing data were $5.8 \%, 54 \%$, and $40 \%$ in definite SSD, and $40 \%, 16 \%$, and $44 \%$ in matched MD, respectively, which shows a similar proportion of missing data but clearly greater ineffectiveness in definite SSD. Namely, substitution of missing data narrowed the difference between the two groups, but a significant difference was observed so that we consider this factor as a characteristic of SSD. Finally, because responses of SSD patients may change on a daily basis, such patients should receive repeated evaluations.

\section{Conclusion}

This is the first study to develop a clinical diagnostic score (A-MUPS score) that differentiates patients with SSD from those with MD for complaints of non-acute pain. We expect the model will help to diagnose patients with SSD in consort with other validations. Further studies are needed to investigate the clinical features of SSD other than complaints of pain, the distinction between SSD and FSS, and the clinical differences among SSD, FSS, and other mental disorder.

\section{Acknowledgment}

The authors would like to thank Editage for the English language editing.

\section{Disclosure}

The authors report no conflicts of interest in this work.

\section{References}

1. Dimsdale JE, Creed F, Escobar J, et al. Somatic symptom disorder: an important change in DSM. J Psychosom Res. 2013;75:223-228.

2. American Psychiatric Association. Diagnostic and Statistical Manual of Mental Disorders. 5th ed. Arlington, VA: American Psychiatric Association; 2013.

3. Frances A. The new somatic symptom disorder in DSM-5 risks mislabeling many people as mentally ill. BMJ. 2013;346:f1580.

4. Lowe B, Spitzer RL, Williams JB, Mussell M, Schellberg D, Kroenke K. Depression, anxiety and somatization in primary care: syndrome overlap and functional impairment. Gen Hosp Psychiatry. 2008;30:191-199.
5. Dimsdale JE, Dantzer R. A biological substrate for somatoform disorders: importance of pathophysiology. Psychosom Med. 2007;69(9):850-854.

6. Bensing JM, Verhaak PF. Somatisation: a joint responsibility of doctor and patient. Lancet. 2006;367:452-454.

7. Biderman A, Yeheskel A, Herman J. Somatic fixation: the harm of healing. Soc Sci Med. 2003;56:1135-1138.

8. Freidl M, Spitzl SP, Prause W, et al. The stigma of mental illness: anticipation and attitudes among patients with epileptic, dissociative or somatoform pain disorder. Int Rev Psychiatry. 2007;19:123-129.

9. Häuser W, Bialas P, Welsch K, Wolfe F. Construct validity and clinical utility of current research criteria of DSM-5 somatic symptom disorder diagnosis in patients with fibromyalgia syndrome. J Psychosom Res. 2015;78(6):546-552.

10. Dimsdale JE, Levenson J. Diagnosis of somatic symptom disorder requires clinical judgment. $J$ Psychosom Res. 2013;75(6):588.

11. Wessely S, Nimnuan C, Sharpe M. Functional somatic syndromes: one or many? Lancet. 1999;354:936-939.

12. Simon GE, VonKorff M, Piccinelli M, Fullerton C, Ormel J. An international study of the relation between somatic symptoms and depression. N Engl J Med. 1999;341(18):1329-1335.

13. Noyes R Jr, Stuart SP, Watson DB. A reconceptualization of the somatoform disorders. Psychosomatics. 2008;49(1):14-22.

14. Fink P, Steen Hansen M, Sondergaard L. Somatoform disorders among first-time referrals to a neurology service. Psychosomatics. 2005;46(6):540-548.

15. Kapur BM, Lala PK, Shaw JL. Pharmacogenetics of chronic pain management. Clin Biochem. 2014; 47(13-14):1169-1187.

16. Colloca L, Klinger R, Flor H, Bingel U. Placebo analgesia: psychological and neurobiological mechanisms. Pain. 2013;154(4):511-514.

17. Klinger R, Colloca L, Bingel U, Flor H. Placebo analgesia: clinical applications. Pain. 2014;155(6):1055-1058.

18. Lowe B, Mundt C, Herzog W, et al. Validity of current somatoform disorder diagnoses: perspectives for classification in DSM-V and ICD-11. Psychopathology. 2008;41(1):4-9.

19. Lieb R, Zimmermann P, Friis RH, Hofler M, Tholen S, Wittchen HU. The natural course of DSM-IV somatoform disorders and syndromes among adolescents and young adults: a prospective-longitudinal community study. Eur Psychiatry. 2002;17(6):321-331.

20. Lieb R, Meinischmidt G, Araya R. Epidemiology of the association between somatoform disorders and anxiety and depressive disorders: an update. Psychosom Med. 2007;69(9):860-863.

21. Noyes R, Stuart S, Watson DB, Langbehn DR. Distinguishing between hypochondriasis and somatization disorder: a review of the existing literature. Psychother Psychosom. 2006;75(5):270-281.

22. Kurlansik SL, Maffei MS. Somatic symptom disorder. Am Fam Physician. 2016;93(1):49-54.

23. Eisenberger NI, Lieberman MD, Williams KD. Does rejection hurt? An FMRI study of social exclusion. Science. 2003;302(5643):290-292.

24. Sharpe M. Somatic symptoms: beyond 'medically unexplained'. $B r J$ Psychiatry. 2013;203(5):320-321.

25. Tracey I. Imaging pain. Br J Anaesth. 2008;101(1):32-39.

26. Yoshino A, Okamoto Y, Yoshimura S, et al. Distinctive neural responses to pain stimuli during induced sadness in patients with somatoform pain disorder: an fMRI study. Neuroimage Clin. 2013;2:782-789.

27. Barr WB. Differential diagnosis of psychological factors evoked by pain presentations. Clin Neuropsychol. 2013;27(1):17-29. 


\section{Supplementary material}

Table SI Final diagnoses of medical diseases according to pain location

\begin{tabular}{|c|c|c|}
\hline Whole body & & \\
\hline Polymyalgia rheumatica & Spondyloarthritis & Myopathy \\
\hline Rheumatoid arthritis & Psoriasis & Steroid \\
\hline Systemic lupus erythematosus & Reactive arthritis & Alcohol \\
\hline Behcet's disease & Undifferentiated & Viral infection \\
\hline Granulomatous polyarteritis & Periodic paralysis & Parvo virus \\
\hline ACTH deficiency & Muscle cramp & Neuropathy \\
\hline Adrenal insufficiency & Lymphoproliferative disease & Diabetic \\
\hline Osteomalacia & Rhabdomyolysis & \\
\hline \multicolumn{3}{|l|}{ Head and neck } \\
\hline Migraine & Malignancy & Trauma \\
\hline Cluster headache & Brain & Epilepsy \\
\hline Paroxysmal hemicrania & Maxillary sinus & Giant cell arteritis \\
\hline Primary stabbing headache & Hypopharyngeal & Relapsing polychondritis \\
\hline Trigeminal neuralgia & Metastasis & PFAPA syndrome \\
\hline Primary headache associated with sexual activity & Dry eye & Kikuchi's disease \\
\hline Occipital neuralgia & Glossitis & Crowned dens syndrome \\
\hline Mental neuralgia & Viral infection & Carotidynia \\
\hline Postherpetic neuralgia & HIV & Subacute thyroiditis \\
\hline Zoster & Pheochromocytoma & Sinusitis \\
\hline Sleep apnea syndrome & Spondyloarthritis & Scleritis \\
\hline Medication overuse headache & Psoriasis & Dyskinesia \\
\hline Meningitis carcinomatosa & Spondylosis & Parkinson's disease \\
\hline Benign tumor & Lichen planus & Syringomyelia \\
\hline \multicolumn{3}{|l|}{ Chest } \\
\hline Angina pectoris & Eosinophilic gastritis & Viral infection \\
\hline Aortic dissection & Intercostal neuralgia & HIV \\
\hline Rupture of Valsalva sinus & Postherpetic neuralgia & Bornholm disease \\
\hline Takayasu arteritis & Costochondritis & Mondor's disease \\
\hline Systemic lupus erythematosus & Gastric ulcer & Drug-induced myalgia \\
\hline SAPHO syndrome & Malignancy & Fracture \\
\hline Amyloidosis & Metastasis & Osteomalacia \\
\hline Familial Mediterranean fever & Lymphoma & Muscle cramp \\
\hline Sarcoidosis & Multiple myeloma & Neuropathy \\
\hline Spondyloarthritis & Lung & Diabetic \\
\hline Reactive arthritis & Gastric & Subcutaneous abscess \\
\hline Ankylosing spondylitis & Benign tumor & Pneumothorax \\
\hline \multicolumn{3}{|l|}{ Undifferentiated } \\
\hline \multicolumn{3}{|l|}{ Abdomen } \\
\hline Gastric ulcer & Idiopathic orchialgia & Familial Mediterranean fever \\
\hline Appendicitis & Mesenteric panniculitis & Amyloidosis \\
\hline Diverticulitis & Retroperitoneal fibrosis & ACNES \\
\hline Invagination & Malignancy & Cholinergic urticaria \\
\hline Cholecystolithiasis & Gastric & Immunoglobulin A vasculitis \\
\hline Chronic pancreatitis & Duodenum & Superior mesenteric artery syndrome \\
\hline Ovulation pain & Small intestine & Angina intestinalis \\
\hline lleus & Colon & Discitis \\
\hline Eosinophilic gastroenteritis & Biliary tract & Radiculopathy \\
\hline Crohn's disease & Pancreas & Intercostal neuralgia \\
\hline Constipation & Ovary & Chronic urticaria \\
\hline Renal abscess & Uterine body & Slipping rib syndrome \\
\hline Endometriosis & Kidney & Thrombophlebitis \\
\hline
\end{tabular}


Table SI (Continued)

\begin{tabular}{|c|c|c|}
\hline \multicolumn{3}{|l|}{ Abdomen } \\
\hline Hernia & Malignant lymphoma & Pubic symphysis inflammation \\
\hline Inguinal hernia & Spinal cord & Zoster \\
\hline Abdominal incision & Metastasis & Trauma \\
\hline Salpingitis & Sarcoidosis & Neuropathy \\
\hline Pelvic inflammatory disease & Systemic lupus erythematosus & Diabetic \\
\hline \multicolumn{3}{|l|}{ Back } \\
\hline Spondylosis & Spinal canal stenosis & Chronic pancreatitis \\
\hline Radiculopathy & Pneumothorax & Malignancy \\
\hline Fracture & Drug-induced myalgia & Metastasis \\
\hline Intercostal neuralgia & Tight filum terminale & Pancreas \\
\hline Discitis & Osteomalacia & Multiple myeloma \\
\hline Costochondritis & SAPHO syndrome & Malignant lymphoma \\
\hline Postherpetic neuralgia & Spondyloarthritis & Lung \\
\hline Bornholm disease & Reactive arthritis & Pleural mesothelioma \\
\hline Viral infection & Ankylosing spondylitis & Gastric \\
\hline Cytomegalovirus & Amyloidosis & Unknown primary \\
\hline Myositis & Systemic lupus erythematosus & Neuropathy \\
\hline Streptococcus & Polymyalgia rheumatica & Diabetic \\
\hline Myopathy & Familial Mediterranean fever & Parkinson's disease \\
\hline Steroid & Cholinergic urticaria & Gastric ulcer \\
\hline \multicolumn{3}{|c|}{ Ossification of the ligamentum flavum } \\
\hline \multicolumn{3}{|l|}{ Upper limb } \\
\hline Rheumatoid arthritis & Frozen shoulder & Plexopathy \\
\hline Palindromic rheumatism & Gout & Diabetic \\
\hline Polymyalgia rheumatica & Pseudogout & Lateral epicondylitis \\
\hline RS3PE & Trigger finger & CIDP \\
\hline Mixed connective tissue disease & Painful arc & Complex regional pain syndrome \\
\hline Antiphospholipid syndrome & Cubital tunnel syndrome & Angioedema with eosinophilia \\
\hline Behcet's disease & Radiculopathy & Lymphoadenitis \\
\hline Dermatomyositis & Syringomyelia & Tuberculosis \\
\hline Systemic lupus erythematosus & Ossification of posterior longitudinal ligament & Kikuchi's disease \\
\hline Sjogren's syndrome & Myelopathy & Parkinson's disease \\
\hline Scleroderma & Glomangioma & ACTH deficiency \\
\hline Graves' disease & Wartenberg's syndrome & Hypopituitarism \\
\hline Familial Mediterranean fever & Frostbite & Adrenal insufficiency \\
\hline Spondyloarthritis & Drug-induced myalgia & Malignancy \\
\hline Psoriasis & Rotator cuff tear & Lung (Pancoast tumor) \\
\hline Inflammatory bowel disease & Myopathy & Malignant lymphoma \\
\hline Reactive arthritis & Hypokalemia & Multiple myeloma \\
\hline Undifferentiated & Viral infection & Myelodysplastic syndrome \\
\hline Osteoarthritis & Parvo virus & Angina pectoris \\
\hline Carpal tunnel syndrome & Cytomegalovirus & Raynaud syndrome \\
\hline Calcific tendinitis & Neuropathy & Thoracic outlet syndrome \\
\hline Tenosynovitis & Diabetic & Neuralgia \\
\hline \multicolumn{3}{|l|}{ Lower limb } \\
\hline Radiculopathy & Baker's cyst & Postherpetic neuralgia \\
\hline Pubic symphysis inflammation & Femur head necrosis & Neuropathy \\
\hline Spinal canal stenosis & Stasis dermatitis & Alcoholic \\
\hline Metatarsalgia & Livedo reticularis & Diabetic \\
\hline Epiphysiopathy & Angioedema with eosinophilia & Vitamin BI deficiency \\
\hline Tenosynovitis & Eosinophilic fasciitis & CIDP \\
\hline Piriformis syndrome & Spondyloarthritis & Restless legs syndrome \\
\hline Osteochondritis dissecans & Reactive arthritis & Complex regional pain syndrome \\
\hline Varicosis & Psoriasis & Erythromegalgia \\
\hline Bursitis & Ankylosing spondylitis & ACTH deficiency \\
\hline Myositis & Undifferentiated & Hypopituitarism \\
\hline Streptococcus & Rheumatoid arthritis & Adrenal insufficiency \\
\hline
\end{tabular}


Table SI (Continued)

\begin{tabular}{lll}
\hline Lower limb & & \\
\hline Myopathy & Palindromic rheumatism & Painless thyroiditis \\
Hypothyroid & Mixed connective tissue disease & Osteomalacia \\
Tarsal tunnel syndrome & Familial Mediterranean fever & Peripheral artery disease \\
Ossification of posterior longitudinal ligament & Dermatomyositis & Raynaud syndrome \\
Erythema nodosum & Polymyositis & Malignancy \\
Morton's disease & Focal myositis & Chondrosarcoma \\
Meniscus injury & Behcet's disease & Uterine cervix \\
Panniculitis & Systemic lupus erythematosus & Multiple myeloma \\
Viral infection & Immunoglobulin A vasculitis & Malignant lymphoma \\
Parvo virus & Cryoglobulinemia vasculitis & Metastasis \\
Cytomegalovirus & Polyarteritis nodosa & Benign tumor \\
Plantar fasciitis & Polymyalgia rheumatica & Myelodysplastic syndrome \\
Gout & RS3PE & Hernia \\
Pseudogout & Sjogren's syndrome & Obturator \\
Neuralgia & Graves' disease & Inguinal \\
Obturator & Muscle cramp & Lymphadenitis \\
Lateral femoral cutaneous nerve & Parkinson's disease & Frostbite \\
\hline Genital area & & \\
\hline Malignancy & Hemorrhoid & Pudendal neuralgia \\
Bladder, ureter & Familial Mediterranean fever & Adhesive arachnoiditis \\
Cholinergic urticaria & Retroperitoneal fibrosis & \\
\hline Abreviations:ACTH, a &
\end{tabular}

Abbreviations: ACTH, adrenocorticotropic hormone; PFAPA, periodic fever, aphthous stomatitis, pharyngitis, and adenitis; SAPHO, synovitis, acne, pustulosis, hyperostosis, and osteitis; ACNES, abdominal cutaneous nerve entrapment syndrome; RS3PE, remitting seronegative symmetrical synovitis with pitting edema; CIDP, chronic inflammatory demyelinating polyneuropathy.

\section{Publish your work in this journal}

The Journal of Pain Research is an international, peer reviewed, open access, online journal that welcomes laboratory and clinical findings in the fields of pain research and the prevention and management of pain. Original research, reviews, symposium reports, hypothesis formation and commentaries are all considered for publication.

\section{Dovepress}

The manuscript management system is completely online and includes a very quick and fair peer-review system, which is all easy to use. Visit http://www.dovepress.com/testimonials.php to read real quotes from published authors. 